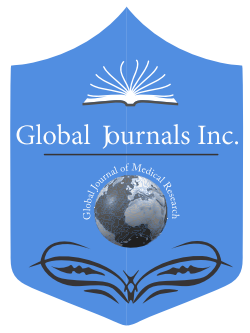

\title{
Liver Damage in Pediatric Critically Ill COVID-19 Patients: Brazilian Case-Series
}

By Michele Luglio, MD, UenisTannuri, MD, PhD, WertherBrunow de Carvalho, MD, PhD, Maria Fernanda Badue Pereira, MD, PhD, Isadora Souza Rodrigues, MD, Cintia Johnston, PhD \& Artur Figueiredo Delgado, MD, PhD

Abstract- Coronavirus disease 2019 (COVID-19) has become an important cause critical care admission worldwide. In the context of newly described multisystem inflammatory syndrome temporally related to SARS-CoV-2 (PIM-TS), the question of liver compromise came into evidence. Our group summarized a case series of 6 critically ill COVID-19 pediatric patients that presented some degree of liver damage, as demonstrated by liver and/or canalicular enzymes elevation, a yet not fully explored characteristic of the infection in the pediatric patient, that may indicate a more severe progression. Observations regarding the role of systemic inflammatory response can be taken from the described cases.

Keywords: hepatic, intensive care, coronavirus.

GJMR-F Classification: NLMC Code: WS 205

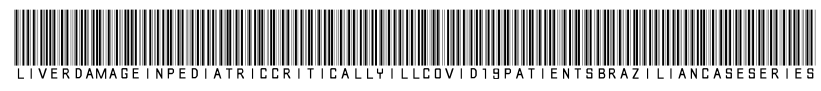

Strictly as per the compliance and regulations of:

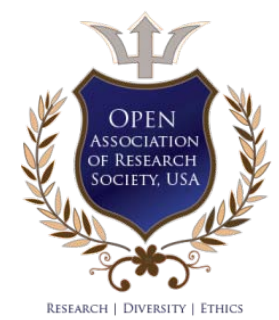

(c) 2020. Michele Luglio, MD, UenisTannuri, MD, PhD, WertherBrunow de Carvalho, MD, PhD, Maria Fernanda Badue Pereira, MD, PhD, Isadora Souza Rodrigues, MD, Cintia Johnston, PhD \& Artur Figueiredo Delgado, MD, PhD. This is a research/review paper, distributed under the terms of the Creative Commons Attribution-Noncommercial 3.0 Unported License http://creativecommons.org/licenses/by-nc/3.0/), permitting all non-commercial use, distribution, and reproduction in any medium, provided the original work is properly cited. 


\title{
Liver Damage in Pediatric Critically III COVID-19 Patients: Brazilian Case-Series
}

\author{
Michele Luglio, $\mathrm{MD}^{\alpha}$, UenisTannuri, $\mathrm{MD}, \mathrm{PhD}^{\circ}$, WertherBrunow de Carvalho, $\mathrm{MD}, \mathrm{PhD}^{\mathrm{p}}$, \\ Maria Fernanda Badue Pereira, $\mathrm{MD}, \mathrm{PhD}^{\omega}$, Isadora Souza Rodrigues, $\mathrm{MD}^{*}$, Cintia Johnston, $\mathrm{PhD}^{\S}$ \\ \& Artur Figueiredo Delgado, $\mathrm{MD}, \mathrm{PhD}^{\mathrm{x}}$
}

Abstract- Coronavirus disease 2019 (COVID-19) has become an important cause critical care admission worldwide. In the context of newly described multisystem inflammatory syndrome temporally related to SARS-CoV-2 (PIM-TS), the question of liver compromise came into evidence. Our group summarized a case series of 6 critically ill COVID-19 pediatric patients that presented some degree of liver damage, as demonstrated by liver and/or canalicular enzymes elevation, a yet not fully explored characteristic of the infection in the pediatric patient, that may indicate a more severe progression. Observations regarding the role of systemic inflammatory response can be taken from the described cases.

Keywords: hepatic, intensive care, coronavirus.

\section{INTRODUCTION}

$(1$ oronavirus disease 2019 (COVID-19) has increasingly become an important cause of critical care admission. Some adult studies and case series have focused on the important aspect of extra-pulmonary commitment by Severe Acute Respiratory Syndrome Coronavirus 2 (SARS-CoV-2), with attention to potential liver damage 1 . Between 14 and $53 \%$ of adult patients with COVID-19 showed alanine aminotransferase (ALT) and/or aspartate aminotransferase elevations ${ }^{1}$.

In the context of the recently described multisystem inflammatory syndrome temporally related to SARS-CoV-2 infection (PIM-TS), Whittaker et $a l^{2}$ compiled data of 58 individuals, showing that ALT median levels on the different phenotypic groups varied from 26 (12-141) to 86 (34-129) U/L.

Our group summarized a series of critically ill COVID-19 pediatric patients with hepatic damage (as demonstrated by liver and/or canalicular enzymes elevation), admitted to a Brazilian tertiary hospital Pediatric Intensive Care Unit (PICU), dedicated to cases of SARS-CoV-2 infection.

Author $\alpha ¥ \S \chi$ : Center of Pediatric Critical Care, Instituto da Criança e do Adolescente do Hospital das Clínicas da FMUSP.

Author o: Pediatric Surgeryand Liver Transplant Department, Instituto da Criança e do Adolescente do Hospital das Clínicas da FMUSP.

Author w: Pediatric InfectiousDiseasesDepartment, Instituto da Criança e do Adolescente do Hospital das Clínicas da FMUSP.

Author $¥$ : Unity ofAnestheticSupport, Instituto Central do Hospital das Clínicas da FMUSP.

Corresponding Author $p$ : Center of Pediatric Critical Care, Instituto da Criança e do Adolescente do Hospital das Clínicas da FMUSP, Brazil. e-mail: werther.brunow@hc.fm.usp.br

\section{Methods}

From March to June 2020, 35 patients were admitted to Pediatric COVID-19 dedicated wards and PICU in a single tertiary center in São Paulo. Of those patients, 15 needed intensive care support. All the patients had a confirmed diagnosis of SARS-CoV-2 infection performed by nasopharyngeal reversetranscriptase polymerase chain reaction (RT-PCR), serological tests (IgM and lgG) and/or diagnosis of PIMTS, following the World Health Organization (WHO) criteria ${ }^{2}$.

Demographic, clinical and laboratory data were obtained from medical records by two independent investigators (ML and ISR). After retrospective analysis of 15 critical patients' records, 6 patients without previous hepatic illnesses showed some degree of liver damage and were included in the case series, after thorough discussion among experts and all authors' agreement. As COVID-19 is a new disease, consensus towards the precise definition of liver damage is still lacking $^{3}$. On this case series, the authors defined liver damage by the presence of new elevations of alanine aminotransferase (ALT), aspartate aminotransferase (AST), gama-glutamyl transferase (GGT), alkaline phosphatase (ALP) and/or Total Bilirubin in relation to the patient's baseline values (when available) or the institution's laboratory references, through the hospitalization for critical COVID-19.

Patients consented at the time of hospital admission for the inclusion in a case series, and approval was obtained from the hospital ethics committee for the report of these cases.

\section{Results}

On table 1, main demographic, clinical and liver enzymes characteristics of those included patients are summarized. On Figure 1, the temporal evolution of AST/ALT and GGT levels, during PICU stay, is shown: 


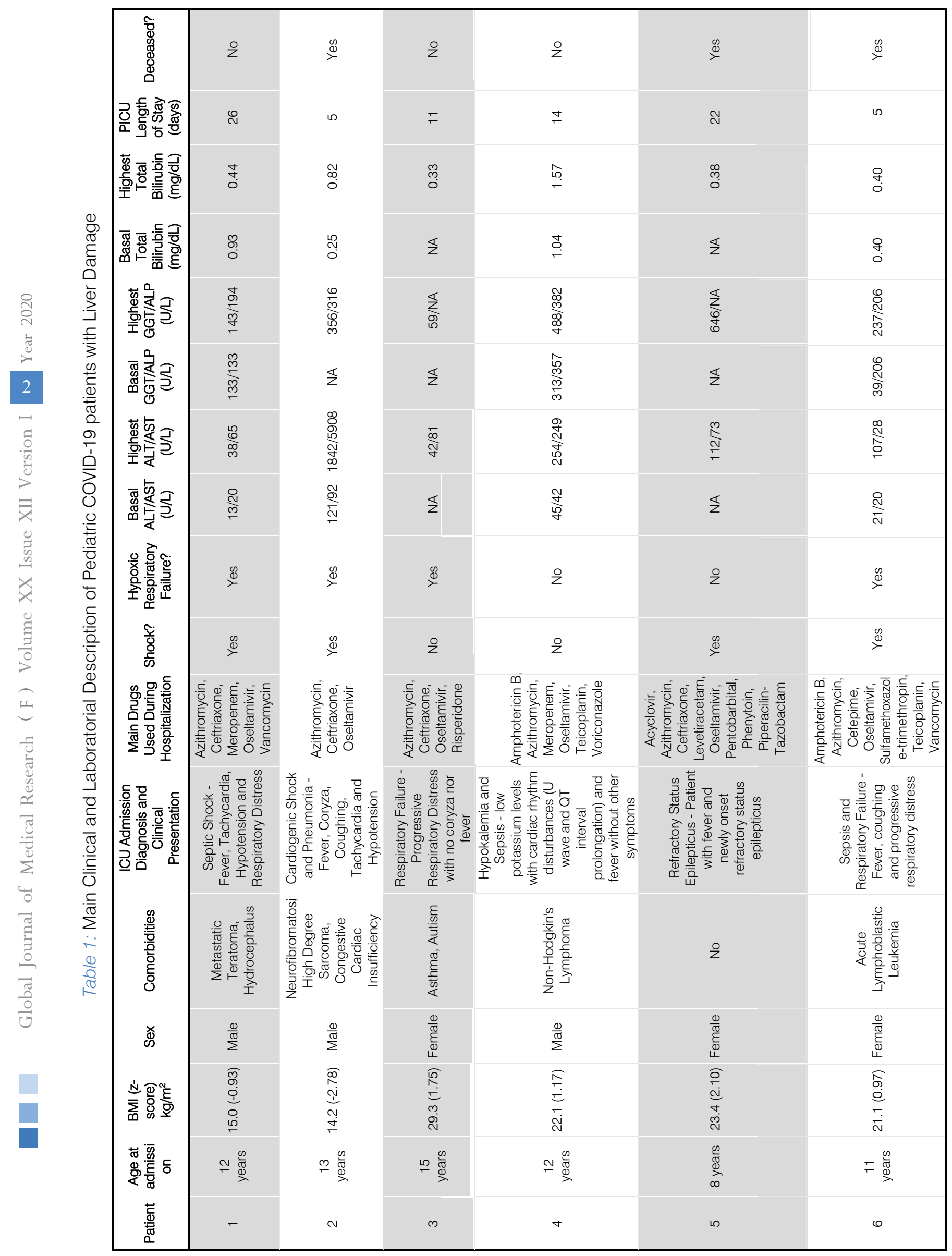


AST

10000

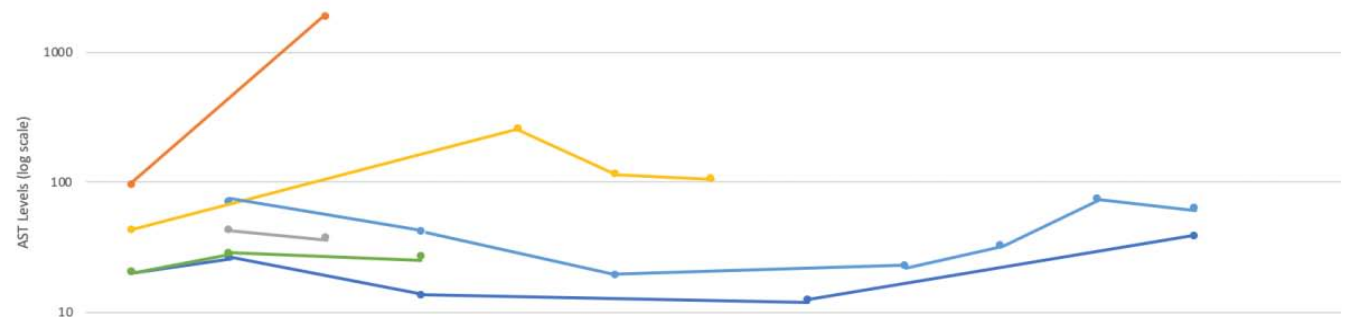

10

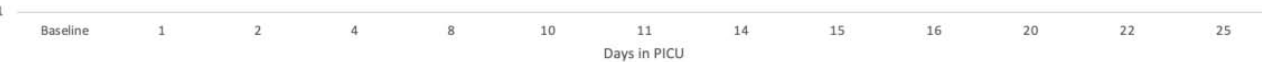

- Patient $1-$ Patient $2-$ Patient $3-$ Patient $4-$ Patient $5 \rightarrow$ Patient 5

A

ALT

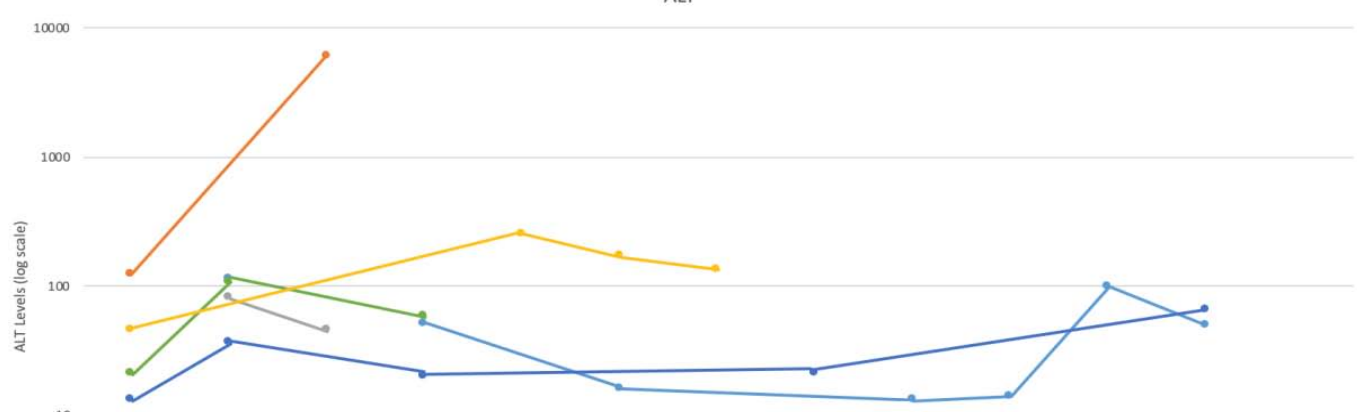

B

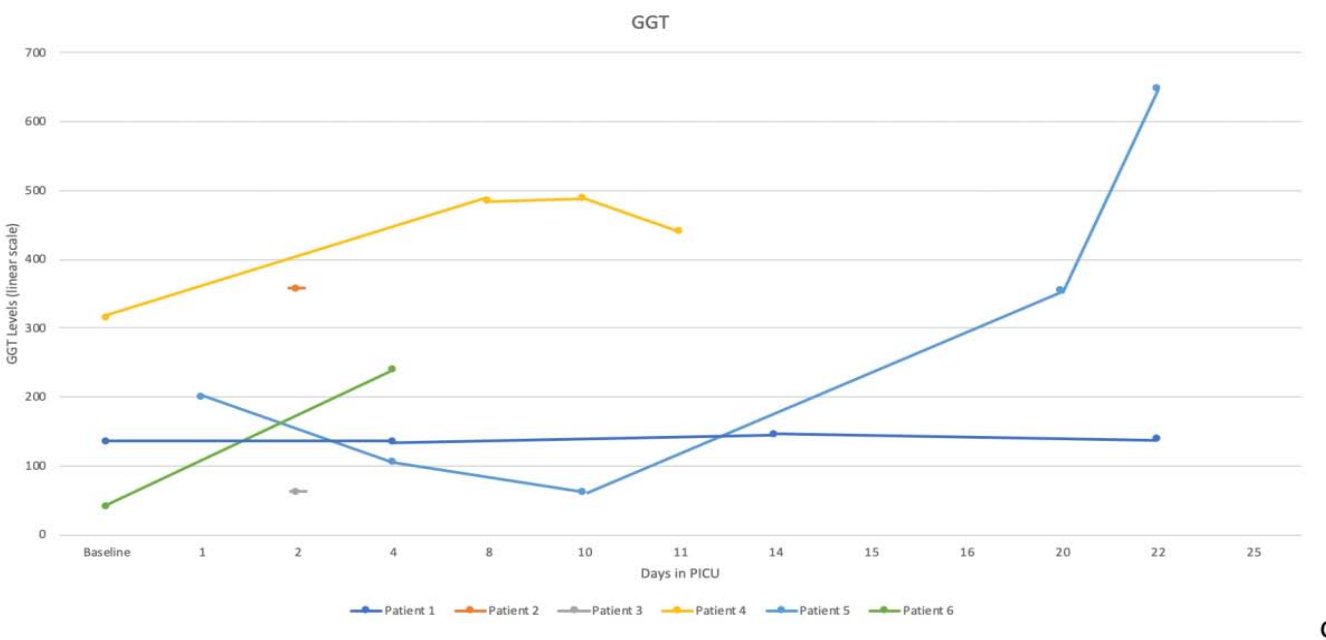

Figure 1: AST, ALT and GGT temporal evolution during PICU stay:

Figure 1: AST, ALT and GGT levels during PICU stay (A: AST levels on log scale vs days in PICU; B: ALT levels on log scale vs days in PICU; C: GGT levels on linear scale vs days in PICU). 
Patient 1:

Admitted at Pediatric Emergency Department (PED) with 5 days of cough and 2 days of progressive respiratory distress and fever. Patient evolved with hypoperfusion and hypotension, managed with the administration of continuous epinephrine. The patient presented no gastrointestinal symptoms. SARS-CoV-2 nasopharyngeal polymerase chain reaction (PCR) was positive, and all other etiologic exams (blood cultures, respiratory virus panel) were negative. Abdominal ultrasound (US) showed normal-sized, regular shaped liver with no intrahepatic biliary channel dilation. Echocardiography showed dilated Left Coronary Artery (z-score $=+2.0)$ and left ventricular systolic and diastolic dysfunction. CRP (318.1 mg/L), Troponin-t (0.092 $\mathrm{ng} / \mathrm{mL})$, CK-MB $(6.23 \mathrm{ng} / \mathrm{mL})$, ferritin $(1,017$ $\mathrm{ng} / \mathrm{mL})$ and D-dimer $(19,514 \mathrm{ng} / \mathrm{mL})$ were elevated at admission and during PICU stay. Prothrombin time (PT) and Activated Partial Thromboplastin time (aPTT) showed elevations, with values of 20.6s and 38.2s (INR $=1.69$ and $R=1.33$ ), respectively. The AST/ALT alterations shown completely resolved after discharge.

\section{Patient 2:}

Admitted to PED with 2 days of weakness and inappetence, followed by cough and progressive respiratory distress. The only gastrointestinal symptoms present during hospitalization were sporadic vomits. The patient showed signs of hypoperfusion and hypotension, with point-of-care ultrasound (POCUS) showing turgid inferior vena cava, right atrium dilation and compromised global systolic function, and Dobutamine infusion was started. SARS-CoV-2 nasopharyngeal PCR was positive, with all other etiologic exams (blood cultures, respiratory virus panel) negative. CRP was elevated on admission (111.0 $\mathrm{mg} / \mathrm{dL}$ ). The patient was transferred to PICU, where evolved with worsening respiratory failure and the need of invasive mechanical ventilation. Echocardiography showed Left Coronary Artery dilation (z-score $=+2.7$ ) and left ventricular systolic and diastolic dysfunction (LVEF $=24 \%$ with Milrinone and Adrenaline). Due to the diagnosis of incomplete Kawasaki disease, two doses of IVIG (total $2 \mathrm{~g} / \mathrm{kg}$ ) were administered and high dose aspirin was started. PT and aPTT showed elevations, with values of 34.9 s and 34.2 s $(\mathrm{INR}=2.86$ and $\mathrm{R}=$ 1.19), respectively. After 5 days in critical care, the patient died.

Patient 3:

Admitted to PED with 4 days of progressive respiratory distress with no fever or other symptoms. No gastrointestinal symptoms were present during hospitalization. Patient was intubated and mechanically ventilated due to hypoxic respiratory failure. SARS-CoV2 nasopharyngeal RT-PCR was positive, with all other etiologic exams (blood cultures) negative. Echocardiography was normal. CRP (48.2 mg/L),
Troponin-t (0.059 ng/mL), d-dimer $(4,157 \mathrm{ng} / \mathrm{mL})$ and $\mathrm{LDH}(892 \mathrm{U} / \mathrm{L})$ were elevated at admission. PT (12.3s, $\mathrm{INR}=1.01)$ and $\mathrm{aPTT}(26.7 \mathrm{~s}, \mathrm{R}=0.98)$ were normal through PICU stay. The patient showed mild ALT/AST elevations, that resolved after hospital discharge. After 11 days on critical care, the patient was discharged to general pediatric ward.

Patient 4:

After 4 months of hospitalization following chemotherapy complications and acute renal failure, the patient began intermittent fever without other symptoms. Infectious screening exams were performed, including SARS-CoV-2 nasopharyngeal RT-PCR that came out positive. Galactomannan test was the only other etiologic test to be positive. Echocardiography, thoracic and cranio-facial CT-scans showed no new alterations. Abdominal CT-scan showed liver dimensions on the upper limit with regular shape and contour, without biliary dilation and mild colonic parietal thickening. In spite of the colonic findings, no gastrointestinal symptoms were present. Patient developed severe hypokalemia and worsening renal function, needing PICU transfer. During the course of SARS-CoV-2 infection, the patient showed a nearly 6-fold ALT/AST elevation, associated with moderate GGT/ALP elevations (in relation to the previous basal values, shown in table 1) and stable values of PT and aPTT, $14.4 \mathrm{~s}(\mathrm{INR}=1.18)$ and $29.2 \mathrm{~s}(\mathrm{R}=1.07)$ respectively. Alterations on liver enzymes resolved after discharge.

\section{Patient 5:}

Admitted to PICU after 4 days of odynophagia, fever and headache, evolving with vomits and episodes of convulsion. The diagnosis of refractory status epilepticus was made, and continuous midazolam and pentobarbital were started after orotracheal intubation. Multiple antimicrobial schemes were used through hospitalization (Table 1). Cerebral Spinal Fluid (CSF) showed mild alterations (3 cells, with normal glucose and protein levels). Cranial Computed Tomography scan (CT-scan) was normal and thoracic CT-scan showed bilateral ground-glass opacifications. SARSCoV-2 PCR was negative, as were all other etiologic tests (CSF culture and viral PCRs, blood cultures, respiratory virus panel). Echocardiography showed right (z-score: +3.0) and anterior descendent (z-score: +2.6) coronary arteries dilation. CRP (318.1 mg/L) and Ddimer $(19,514 \mathrm{ng} / \mathrm{mL})$ were elevated at admission and during intensive care. IVIG and high-dose methylprednisolone pulse-therapy were administered, due to the diagnosis of PIM-TS. The patient presented episodes of melena and was submitted to endoscopic evaluation that showed two ulcerations on duodenal superior wall. PT also showed mild elevations, with a peak of 15.5s (INR $=1.27$ ) while aPTT was normal throughout the PICU stay. The patient developed progressive metabolic disturbances and uncontrolled 
status epilepticus, dying of refractory shock after 22 days in PICU.

Patient 6:

Patient on the seventh day after chemotherapy (vincristine and doxorubicin) was admitted to the PED with 2 days of fever, cough and progressive respiratory distress. The patient was diagnosed with neutropenia and sepsis, starting empiric antimicrobial therapy, with association of Amphotericin B through the course of hospitalization. SARS-CoV-2 nasopharyngeal PCR was positive, with all other etiologic exams (blood cultures, respiratory virus panel) negative. The patient evolved with respiratory failure and hemodynamic instability, needing invasive ventilatory and inotropic support on the second day of hospital admission. No gastrointestinal symptoms appeared during PICU stay. D-dimer (1,932 $\mathrm{ng} / \mathrm{mL})$ and ferritin $(3,295 \mathrm{ng} / \mathrm{mL})$ showed elevations through hospitalization, with normal PT (13s, INR = 1.0) and aPTT (30s, $R=1.11$ ). Echocardiography showed small pericardial effusion and thoracic CT-scan showed diffuse bilateral ground-glass opacifications. The patients deceased after 5 days in ICU due to refractory shock.

\section{Discussion}

Important variations are found when evaluating ALT/AST levels in patients with COVID-193,4. This case series corroborates previous findings, with AST elevations ranging from 65 to $5908 \mathrm{U} / \mathrm{L}$.

Transaminase elevations seen in this series may be related to four mechanisms: (1) Drug induced liver injury (DILI); (2) Direct biliary injury by coronavirus; (3) inflammatory response in the context of cytokine storm; (4) Ischemia/Reperfusion and microthrombosis ${ }^{7}$.

Abnormalities on liver enzymes seen can occur on either the initial viremia phase or during the subsequent inflammatory phase ${ }^{7}$. It was already reported that high ALT and bilirubin can be considered biomarkers of a more severe clinical course ${ }^{7,8}$.

The potential for DILI in the context of critical COVID-19 cannot be neglected ${ }^{9}$. All patients included in our case series received at least one Category A or B hepatotoxic drug, as described by LiverTox ${ }^{10}$. Drug induced liver damage may be an important contributing factor to a multifactorial condition.

Different from previous reports ${ }^{4,5}$, patients included showed moderate elevations on GGT levels, consistent with experimental observations that cholangiocytes express ACE-2 receptors, a target for direct viral invasion and damage ${ }^{6}$.

Three patients had features consistent with PIMTS (patients 1, 2 and 5) ${ }^{2}$, as defined by the WHO criteria. SARS-CoV-2 can be considered the trigger of an uncontrolled systemic inflammatory condition or cytokine "storm". In this context, cellular apoptosis and necrosis and the release of damage-related patterns may induce injuries to multiple organs, the liver included. Hepatic endothelial involvement in the inflammatory process, with consequent neutrophil extracellular traps (NETS) stimulation and microthrombi formation, in a process similar to the one happening in the lungs ${ }^{11}$, needs to be further studied.

Effenbergeret $a l^{12}$ explored the connection between systemic inflammation and liver injury in COVID-19 hospitalized patients. IL-6 and CRP levels positively correlated with AST elevations (respectively, $r^{2}$ $=0.481, p<0.001$ and $\left.r^{2}=0.38, p<0.001\right)$ in all 96 included patients with pronounced effects on critically ill patients. Those findings correlate with this case series, as high levels of CRP and PCT were seen in patients with liver enzyme elevations. This cytokine "storm" may play a vital role in the hepatic damage.

In the beginning of the pandemic, the main focus of intensivists was on the viral potential to induce hypoxia. Hypoxia-reperfusion injury to the liver can stimulate hepatocyte cell death and inflammation, marked by oxygen reactive species accumulation ${ }^{13}$, another potential causative mechanism to liver damage.

PICU mortality among the described patients was $50 \%(3 / 6)$ with a length of stay of $12.5(6.5-20)$ days, while the remainder of the pediatric COVID-19 critically ill patients experienced a mortality rate of $33.3 \%$ (3/9) and length of stay of 7 (3-10) days. Presence of liver enzyme alterations indicates a more severe disease course, with all patients but one (patient 4) needing ventilatory, hemodynamic support or both. Given the tertiary condition of our center, the population included is mainly composed of patients with chronic conditions, what have impacts on the outcomes seen. In regard of the liver enzyme elevations, special care was taken to compare previous individual baseline levels to the highest values seen towards disease course.

This study has limitations of a small case series, which needs confirmation on larger groups. Due to the retrospective nature of the study and to conditions inherent of a pandemic in a developing country, a complete evaluation of radiological and histological aspects of the hepatic compromise may be lacking. As the focus was on the clinical description of patients with liver abnormalities, comparison with the global data of all COVID-19 patients admitted to the hospital was not made and can be an important future step.

Reports from over the world ${ }^{13}$ show slightly different outcomes and evolutions of clinical conditions associated with COVID-19 in children. In a recent report by Sadiq et $\mathrm{al}^{14}$, Pakistani children with PIM-TS showed an incidence of coronary artery aneurism (62.5\%), higher than European and North American numbers (9$36 \%$ ). Some of the findings in our case series can be justified by regional differences, that may be better identified in future studies. Knowledge of those disparities are relevant to deepen the understanding of the clinical potential of SARS-CoV-2 infection. 
This may be the first pediatric COVID-19 case series focused on liver damage, an important start-point to raise clinical attention to this aspect of SARS-CoV-2 infection. Further characterization of this population of patients may elucidate some still obscure aspects of COVID-19 related hepatic physiopathology.

Conflict of Interest Disclosures: The authors have no conflict of interest to disclose.

Funding/Support: No funding was secured for this study.

\section{References Références Referencias}

1. Zhang C, Shi L, Wang FS. Liver injury in COVID-19: management and Challenges. Lancet Gastroenterol Hepatol, 2020. [DOI: 10.1016/S2468-1253(20) 30057-1].

2. Whittaker E, Bamford A, Kenny J, et al. Clinical characteristics of 58 children with Pediatric Inflammatory Multisystem Syndrome Temporally Associated with SARS-CoV-2. J Amer Med Assoc, 2020. [DOI: 10.1001/jama.2020.10369].

3. Cai Q, Huang D, Yu H, et al. COVID-19: Abnormal Liver Function Tests. J Hepatol, 2020. [DOI: 10.1016/j.hep.2020.04.006].

4. Guan WJ, Ni ZY, Hu Y, et al. Clinical Characteristics of 2019 novel coronavirus infection in China. N Engl J Med, 2020. [DOI: 10.1056/NEJMoa2002032].

5. Fan Z, Chen L, Li J, et al. Clinical Features of COVID-19-related Liver Functional Abnormality. Clin Gastroenterol Hepatol, 2020. 18(7):1561-1566.

6. Chai $X$, Hu L, Zhang $Y$, et al. Specific ACE2 expression in cholangiocytes may cause liver damage after 2019-nCoV infection. bioRxiv, 2020. [DOI: 10.1101/2020.02.03.931766].

7. Morgan K, Samuel K, Vandeputte M, et al. SARSCoV-2 Infection and the Liver. Pathogens, 2020. [DOI: 10.3390/pathogens9060430].

8. Xu L, Liu J, Lu M, et al. Liver Injury during Highly Pathogenic Human Coronavirus Infections. Liver Int, 2020. 5:428-430.

9. Li Y, Xiao SY. Hepatic Involvement in COVID-19 patients: Pathology, pathogenesis and clinical implications. J Med Virol, 2020. [DOI: 10.1002/ jmv.25973].

10. LiverTox. Available online: http://livertox.nlm.nih.gov (accessed on July $2^{\text {nd }}, 2020$ ).

11. Zuo Y, Yalavarthi S, Shi H, et al. Netrophil extracellular traps in COVID-19. JCl Insight, 2020. 5(11):e138999.

12. Effenberger M, Grander $C$, Grabherr $F$, etl al. Systemic inflammation as fuel for acute liver injury in COVID-19. Digestive and Liver Disease, 2020. [DOI: 10.1016/j.dld.2020.08.004]

13. Zhang XJ, Cheng X, Yan ZZ, et al. An ALOX-12-12HETE-GPR31 signaling axis is a key mediator of hepatic ischemia-reperfusion injury. Nat Med, 2018. 24:73-83.
14. Sadiq M, Aziz OA, Kazmi U, et al. Multisystem inflammatory syndrome associated with COVID-19 in children in Pakistan. Lancet Child Adolesc Health, 2020. [DOI: 10.1016/S2352-4642(2030256-X] 